\title{
An efficient approach toward increasing wireless sensor networks lifetime using novel clustering in fuzzy logic
}

\author{
Morteza Asghari Reykandeh ${ }^{1,}$, Ismaeil Asghari Reykandeh ${ }^{2}$ \\ ${ }^{1}$ Department of Computer Engineering, Islamic Azad University Khoy Branch, Khoy, Iran \\ ${ }^{2}$ Department of Computer Engineering, Islamic Azad University Sari Branch, Sari, Iran
}

Email address:

Asghari_stu@yahoo.com (M. Asghari. R), ismaeil.asghari@gmail.com (I. Asghari. R)

To cite this article:

Morteza Asghari Reykandeh, Ismaeil Asghari Reykandeh. An Efficient Approach Toward Increasing Wireless Sensor Networks Lifetime Using Novel Clustering in Fuzzy Logic. International Journal of Intelligent Information Systems. Special Issue: Research and Practices in Information Systems and Technologies in Developing Countries. Vol. 3, No. 6-1, 2014, pp. 38-44. doi: 10.11648/j.ijiis.s.2014030601.17

\begin{abstract}
Wireless sensor network (WSN) is composed of a large number of sensor nodes that are connected to each other. In order to collect more efficient information, wireless sensor networks are classified into groups. Classification is an efficient way to increase the lifetime of wireless sensor networks. In this network, devices have limited power processing and memory. Due to limited resources in wireless sensor networks, increasing lifetime was always of attention. An efficient routing method is called clustering based routing that finds optimum cluster heads and finding the correct number of them in each cluster remains a challenge. In this paper, we propose a novel and efficient method for clustering using fuzzy logic with four appropriate inputs and combine it with the good features of Low-Energy Adaptive Clustering Hierarchy (LEACH). Simulation results show that our method is more efficient compared to other distributed algorithms, because the proposed method if fully distributed. The result show that compared to centralized, the speed is more and its energy consumption is less.
\end{abstract}

Keywords: Wireless Sensor Networks, Clustering, Cluster Head, Fuzzy Logic, Lifetime

\section{Introduction}

In the past years, increasing improvements in digital electronics, semi-conductors building technology, and wireless communication, have led to develop tools with small size, low price and high power with communicating, computing and tracking capabilities. Wireless Sensor Networks (WSN) consists of hundreds or even thousands of such sensor tools that use radio frequencies to perform its tracking task [1-6]. A WSN consists of a large number of sensor nodes and a Base Station (BS). These sensors collect data and send them to the BS via radio transmitter. They have limited power and computational capacity. WSNs can be used in many applications such as military, biomedical, and environmental applications.

There are various challenges in wireless sensor networks because of its special features. One of these challenges is the nodes limited power supply. In most cases the power supply is irreplaceable and non-rechargeable. So WSNs must use methods that reduce energy consumption of nodes [1,2 and 3].

The data that are sensed by nodes should be transferred to a station for processing and decision making. This station is called base-station or sink.

If each node sends its data directly to the sink a lot of energy is consumed. Since the sensed values by the close nodes are a little different, there is a possibility of redundancy in the transmitted data.

As mentioned before, a lot of energy is consumed for sending data to the sink. As a result, cluster head nodes are faced with the challenge of fast reducing energy. As soon as the cluster head off a part or the entire network falls of work. To avoid this issue a method is to cluster head nodes equipped with replaceable and rechargeable energy source. Another way is continuously change cluster heads among the nodes within the network to decrease the distributed energy consumption in the network [5].

We use the second method in which the nodes are homogeneous. In the proposed method cluster head is selected first. Each node selects then the nearest cluster head to its own. Thus clusters are produced.

Our method is similar to Low-Energy Adaptive Clustering Hierarchy (LEACH) [2].

Two periods of time are repeated alternately until the end of the network: Stage of cluster formation and stage of stability. 
In this structure, similar to $\mathrm{LEACH}$, each node itself decides to be cluster head and makes other nodes aware of this issue. Selecting cluster heads in LEACH are stochastic. While in our, method a cluster head is selected based on several parameters and a fuzzy system.

Because LEACH selects cluster head only with stochastic and does not care to other parameters such as residual energy and location of nodes, it does not necessarily make the best clusters.

For example, a node may be cluster head that have little energy may be turned off or if an isolated node becomes cluster head, other nodes must spend a lot of energy for sending data to this cluster head. Nevertheless, LEACH provides well uniform distribution of cluster heads and has high performance.

We use a fuzzy system with appropriate inputs to overcome the weakness of LEACH. The inputs that we consider in the fuzzy system are: number of neighbors, centrality, energy remaining, frequencies of signals received from neighbors and the number of round that the node wasn't cluster head. These parameters are not so closely related and can easily work with these heterogeneous parameters by using fuzzy logic. Also a fuzzy system does not need much computational complexity, consequently it is suitable for WSN.

The rest of this paper is organized as follows. In the next section, we give an overview of related work and some shortcomings of stochastically selecting cluster-heads. In section 3 we give an overview of fuzzy logic. In section 4 we describe our proposed method. Simulation results are presented in section 5. Finally, section 6 concludes the paper.

\section{Related Works}

A typical WSN architecture is shown in Figure 1. The nodes send data to the respective cluster-heads, which in turn compresses the aggregated data and transmits it to the base station. For a WSN we make the following assumptions:

- The base station is located far from the sensor nodes and is immobile.

- All nodes in the network are homogeneous and energy constrained.

- Symmetric propagation channel.

- Base station performs the cluster-head election.

- Nodes have little or no mobility.

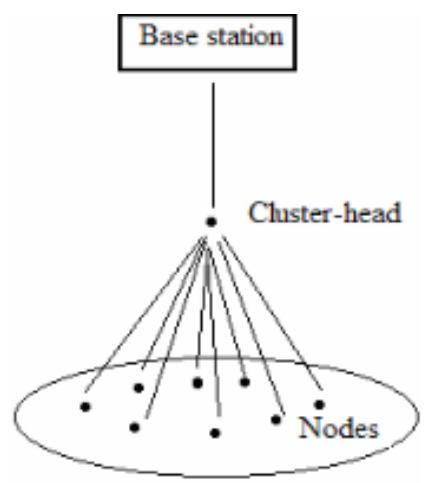

Figure 1. WSN Architecture.
One of the most popular cluster based routing protocol is Low Energy Adaptive Clustering Hierarchy protocol $(\mathrm{LEACH})$ that is proposed in [4]. The operation of LEACH is divided into rounds and each round consists of setup phase and the steady state phase. In the setup phase, the clusters are organized and Cluster Heads (CHs) are selected. Each sensor $\mathrm{n}$ generates a random number between 0 and 1 . If this number is less than $T(n)$ defined by Equation 1, then sensor $n$ would be selected as a cluster head.

$$
T(n)= \begin{cases}\frac{p}{1-p \times\left(r \bmod \frac{1}{p}\right)} & \text { if } n \in G \\ 0 & \text { if } n \notin G\end{cases}
$$

In this equation, ' $\mathrm{p}$ ' is the desired percentage of $\mathrm{CHs}$, ' $\mathrm{r}$ ' specifies the current round and ' $G$ ' is the set of nodes which have not been selected as cluster head in the past $1 / \mathrm{P}$ rounds. Optimal number of cluster heads is estimated to be $5 \%$ of the total number of nodes. After cluster head election, the $\mathrm{CHs}$ broadcast an advertisement message and other nodes select the closest $\mathrm{CHs}$ based on the received signal strength. Although $\mathrm{LEACH}$ is able to increase the network lifetime, but it has two main weaknesses:

- It is possible that no or many of CHs are selected.

- It is possible that too many $\mathrm{CHs}$ are located in a specific area. This means that $\mathrm{CHs}$ are not selected in a distributed manner.

Gupta [6] used fuzzy logic to find cluster heads. In this algorithm three fuzzy variables is used for cluster head selection. Node's energy, node's concentration and node's centrality are these parameters. In this approach, the base station primarily collects the necessary information from all nodes and then selects a node as a cluster head according to the fuzzy rules. In this approach there is only one selected $\mathrm{CH}$ for each round, whereas more $\mathrm{CHs}$ are needed for balancing energy consumption and improving network lifetime.

In [7], Kim offers CHEF in which, the same as Gupta [6], the CHs are selected based on a fuzzy logic. The difference is that in this approach more than one cluster head is selected locally in each round. The fuzzy set includes nodes' energy and their local distances. CHEF also generates a random number for each sensor and if it is less than a predefined threshold, $\mathrm{P}_{\text {opt }}$, then the node's chance is determined. Thus, there may be some qualified nodes that lose their chance on a random manner [7].

The method that is presented in [8] is a fuzzy approach to select cluster heads. This method is centralized and the network is aware of nodes coordinates. The decision for selecting a node as cluster head is done by the sink. This method is based on three variables, node energy remaining, node concentration and centrality which decides about being a node cluster head.

Heinzelman et, al. [9] introduced a method called LEACH-FL. The method uses a fuzzy system with three input battery level, node density and distance from the sink, for selecting cluster heads. This method is introduced with the assumption that the network coordinates is available.

Both of these two methods are centralized. Therefore they 
are not suitable for the environments that required real-time processing. Also a lot of energy is spent for sending nodes situation such as energy remaining to the sink. These methods assumed that the network coordinates is available. For this issue, nodes need to be equipped with additional hardware such as Global Positioning System (GPS), which is not possible in all environments. Another issue is that we can use inputs for fuzzy system more efficient than inputs of these methods fuzzy systems [10].

Based on this discussion, we propose a distributed method where each node itself makes decision about being cluster head or not. This method works in all environments and therefore does not need the coordinates of nodes. This method by choosing suitable inputs for fuzzy system, is more efficient than the existence method and better clusters will be made.

\section{Fuzzy Logic}

Fuzzy logic (FL) is defined as the logic of human thought, which is much less rigid than the calculations computers generally perform. Fuzzy Logic offers several unique features that make it a particularly good alternative for many control problems. It is inherently robust since it does not require precise, noise-free inputs and can be programmed to fail safely $[11,12]$.

Fuzzy sets are described by the range of real values over which the set is mapped, called domain, and the membership function. A membership function assigns a truth (crisp) value between 0 and 1 to each point in the fuzzy set's domain. Depending upon the shape of the membership function, various types of fuzzy sets can be used such as triangular, beta, PI, Gaussian, sigmoid, etc. The trapezoidal and triangular membership functions suitable for real-time operation because they don't make complex computations and also have enough accuracy.

A Fuzzy system basically consists of three parts: fuzzification, inference engine, and defuzzification. Fig. 1 shows the fuzzy system components that we use in this paper. The fuzzifier maps each crisp input value to the corresponding fuzzy sets and thus assigns it a truth value or degree of membership for each fuzzy set. The fuzzified values are processed by the inference engine, which consists of a rule base and various methods for inferring the rules.

The rule base is simply a series of IF-THEN rules that relate the input fuzzy variables with the output fuzzy variables using linguistic variables, each of which is described by a fuzzy set, and fuzzy implication operators such as AND, OR, etc.

The defuzzifier performs defuzzification on the fuzzy solution space. It finds a single crisp output value from the solution fuzzy space. Some of common defuzzification techniques are: Center of Area (COA)[14], Center Of Gravity (COG)[15], Extended Center of Area (ECOA)[14], Mean of Maxima (MeOM)[16]. In this paper we use COA method for defuzzification which is one of the simplest and most widely used methods, The method is fast, because only simple oper tions are used in it, it gives continual change of defuzzification value, hence it is convenient to be used in fuzzy controllers. [17].

\section{Proposed Method}

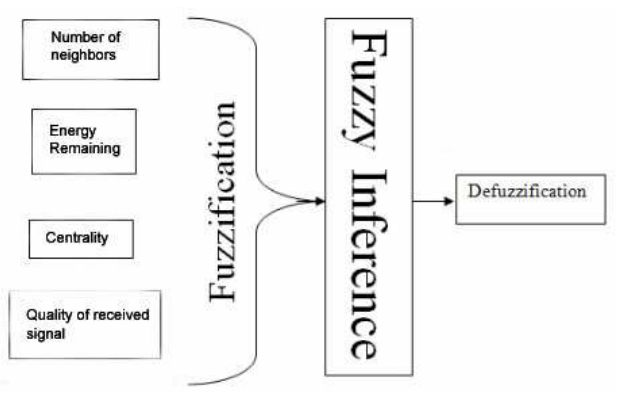

Figure 2. Fuzzy system components

Figure2 depicts the fuzzy system that is used by our method.

The fuzzy system inputs are crisp numbers converted to fuzzy values by membership functions. The nodes simply determine these input values. The nodes are aware of neighbor nodes and the distance to them, as soon as one sends and receiving data. We suppose here, the number of head clusters is 5 percent of total number of sensors in each round.

Parameters that have important role in selecting the head cluster and forming cluster are:

\subsection{Number of Neighbors}

If a node is in center of attention which means many signals pass through its order to reach a cluster head, then it is better that this node becomes cluster head.

$$
\text { Max number of neighbors }=n-1
$$

\subsection{Remaining Energy}

A sensor that has maximum energy level; because the overhead of head cluster is more than other nodes.

$$
E_{T X}(l, d)=\left\{\begin{array}{l}
l * E_{\text {elec }}^{T X}+l * \varepsilon_{f s} * d^{2}, d<d_{0} \\
l * E_{\text {elec }}^{T X}+l * \varepsilon_{m p} * d^{4}, d \geq d_{0}
\end{array}\right.
$$

$E_{R X}(l)=l * E_{\text {elec }}^{R X}$

\subsection{Centrality}

The closest sensor to gravity center of the cluster. Indeed, it is a node that average distance of other nodes in the cluster from it is minimum. Centrality of head cluster, leads to reducing energy consumption for communicating intra-cluster (between member nodes and head cluster).

\subsection{Quality of Received Signals}

This criterion is indeed based on which node receives more signal power; as many signals pass from this node, so it can be a good head cluster.

This operation is done easily by using of Received Signal Strength Indication (RSSI) technique [11].

Membership functions that convert crisp input values to fuzzy values are shown by fig. 3 . 


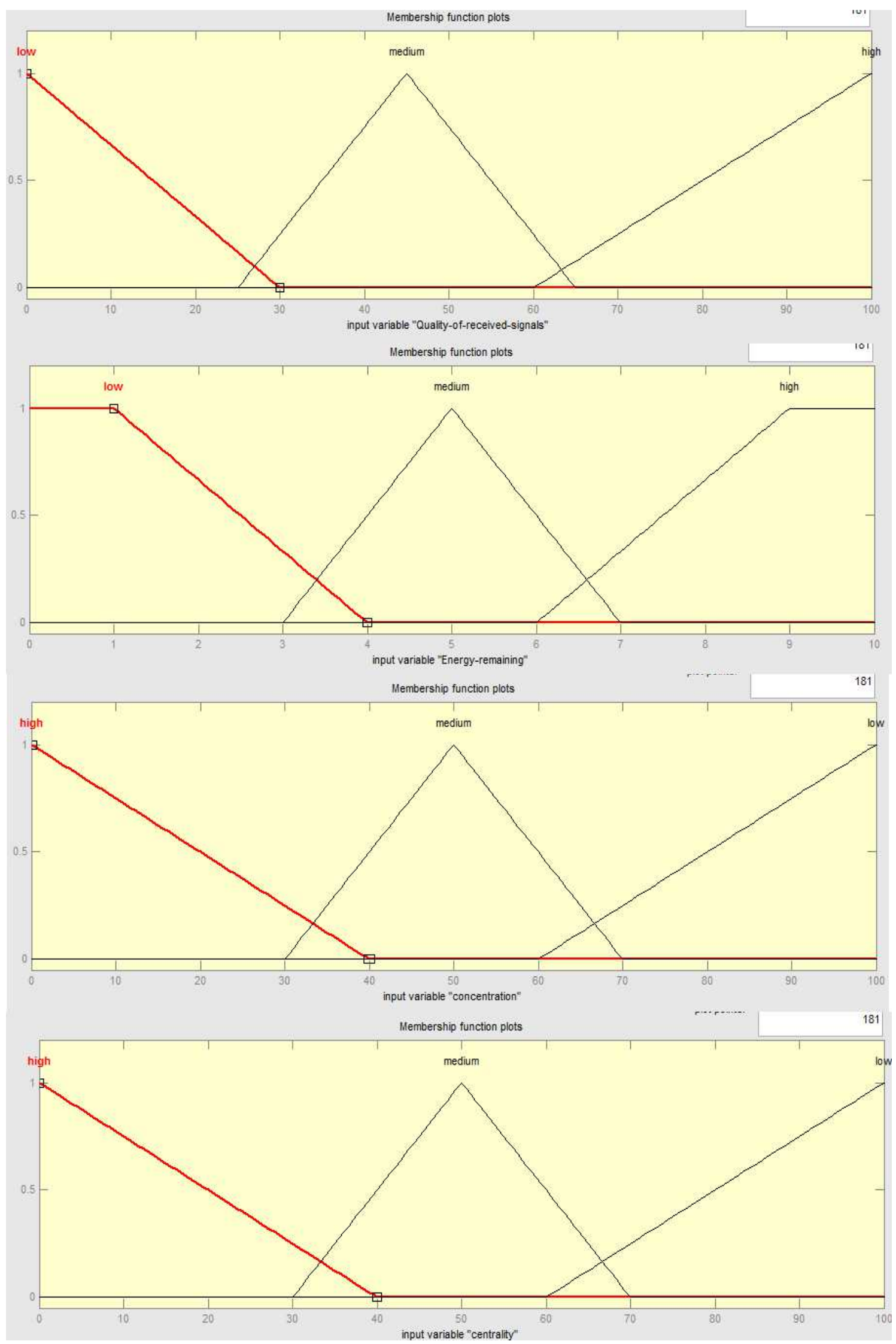

Figure 3. Fuzzy set for fuzzy input variable Number of Neighbors, Remaining Energy, Centrality and Quality of Received Signals

In this stage (fuzzy inference) we use mamdani inference method to determine output from inputs [18]. Some rules that mamdani method employs are listed in Table 1. 
Table 1. Some of the existence rules.

\begin{tabular}{lllll}
\hline Concentration & Energy & Centrality & Quality Signal & Chance \\
\hline low & low & low & low & worth \\
low & low & low & medium & v-low \\
low & low & high & high & low \\
low & medium & low & low & v-low \\
low & medium & high & high & medium \\
low & high & low & low & low \\
medium & medium & high & high & high \\
medium & high & low & low & medium \\
medium & high & high & high & v-high \\
high & low & low & low & medium \\
high & medium & medium & low & high \\
high & medium & medium & medium & high \\
high & medium & medium & high & v-high \\
high & medium & high & low & v-high \\
high & high & high & high & best \\
\hline
\end{tabular}

We need a membership function to convert the obtained output from fuzzy to crisp in the fuzzification stage. This function is shown in fig. 4.

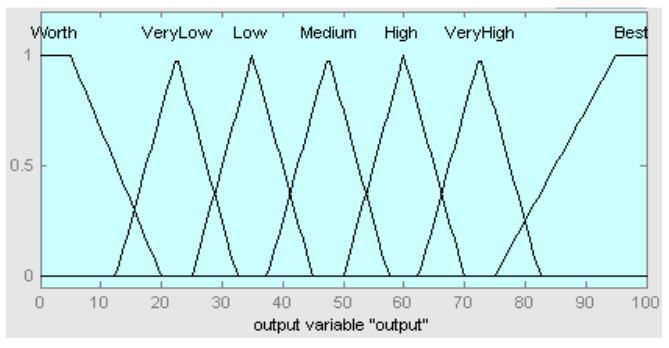

Figure 4. Output membership function.

In this paper, we use the Center of Area (COA) method to deffuzificate output. Threshold $(\alpha)$ in this measure is obtained from Equation 4.

$$
\alpha=\frac{\int_{z} \mu A(x) z d z}{\int_{\mathrm{z}} \mu \mathrm{A}(\mathrm{z}) \mathrm{dz}}
$$

Where $\alpha$ is the non-fuzzy output for the fuzzy system (z) and $\mu \mathrm{A}(\mathrm{Z})$ is aggregated output membership function.

\section{Results and Discussion}

We have used of Matlab [11] for simulations. Fig. 6 shows influence of some fuzzy inputs on the output $(\alpha)$.

To compare with LEACH and LEACH-FL, the networks that have an area of $100 * 100$ with 20 nodes are selected. The coordinate of the BS is $(50,200)$ and the energy parameters are shown in table 2.

Table 2. Energy parameters

\begin{tabular}{llll}
\hline Initiate energy & $\mathbf{0 . 5} \mathbf{J}$ & Eelect & $\mathbf{5 0 n j} / \mathbf{b i t}$ \\
\hline$\varepsilon \mathrm{fs}$ & $10 \mathrm{pj} /(\mathrm{bit} * \mathrm{~m} 2)$ & $\varepsilon a \mathrm{mp}$ & $0.0013 \mathrm{pj} /(\mathrm{bit} * \mathrm{~m} 4)$ \\
\hline
\end{tabular}

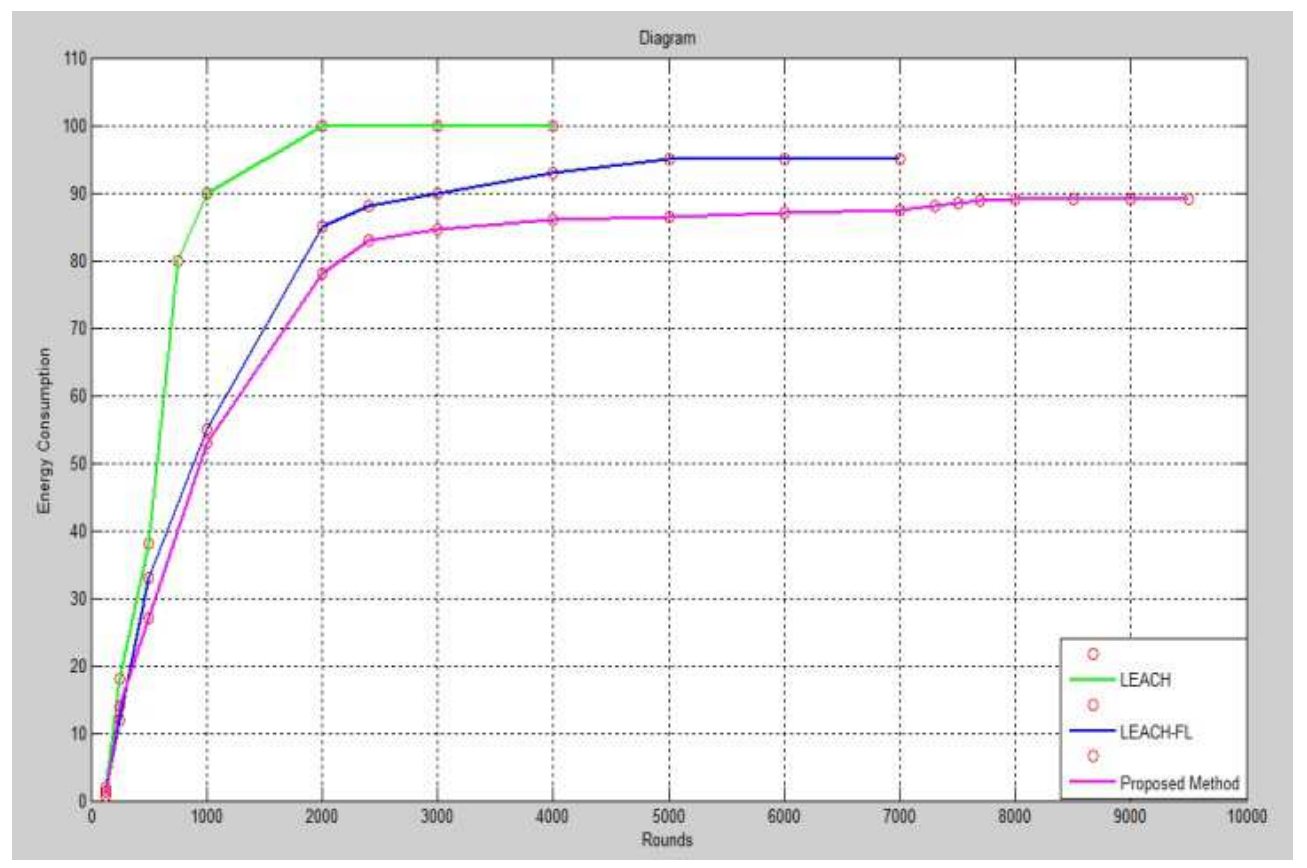

Figure 5. Distribution of energy consumption according to the number of rounds for each algorithm

Fig. 5 shows difference between proposed method and LEACH and LEACH-FL in energy consumption.

Residual energy of network in each round can be a good metric to measure the energy efficiency of the algorithms. The less steep the figure is the more clearness is the energy utilization balance and fairer distribution of energy on the nodes would be.

Consider that the proposed method consumes less energy 
than other two methods and thus the network lifetime increases.
Fig. 6 shows alive nodes in sense of number of rounds in these methods.

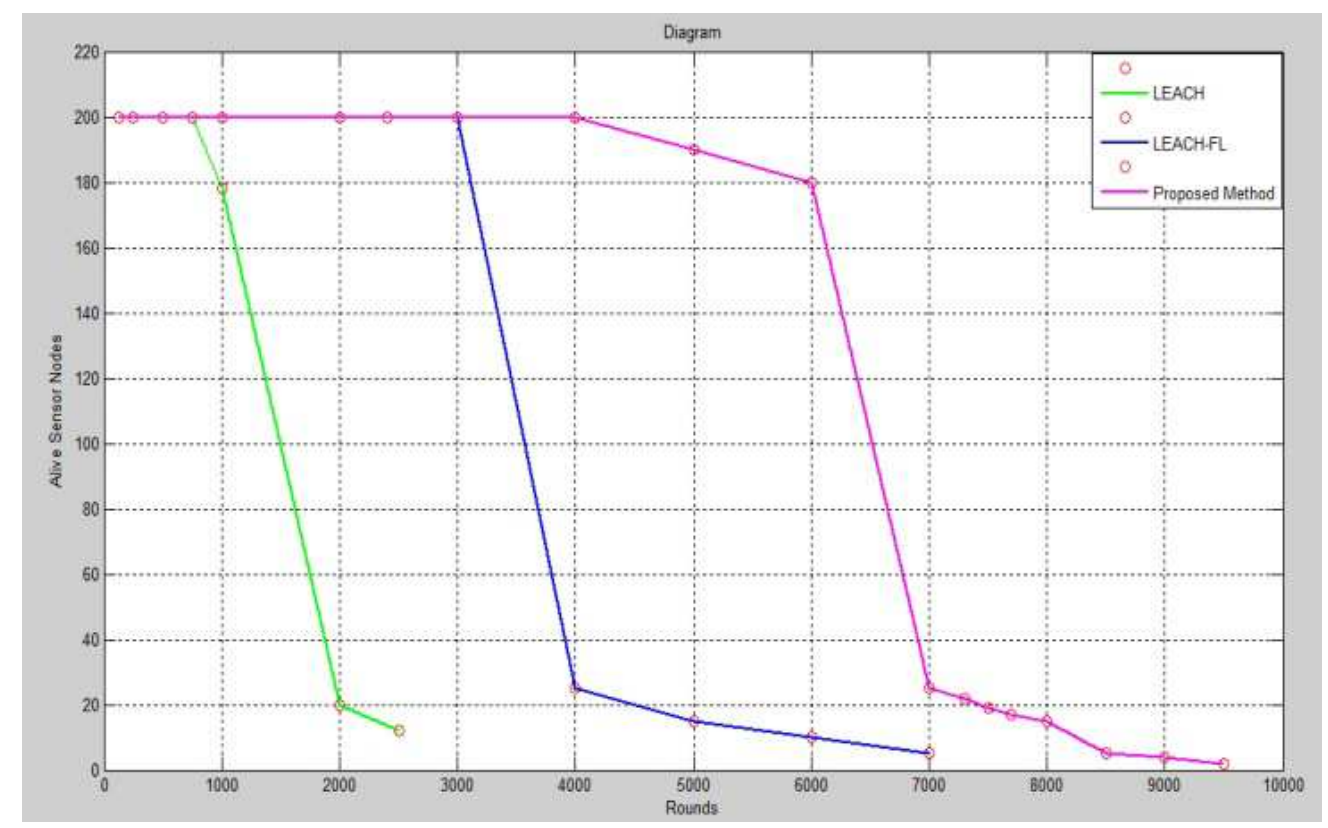

Figure 6. Distribution of alive sensor nodes according to the number of rounds for each algorithm

Unless there are six authors or more give all authors' names; do not use "et al.". Papers that have not been published, even if they have been submitted for publication, should be cited as "unpublished" [4]. Papers that have been accepted for publication should be cited as "in press" [5]. Capitalize only the first word in a paper title, except for proper nouns and element symbols.

For papers published in translation journals, please give the English citation first, followed by the original foreign-language citation [6].

\section{Conclusion}

Our aim of doing this research is to achieve an optimal approach for clustering in Low-Energy Adaptive Clustering Hierarchy (LEACH). Since all operations for cluster formation are done locally a large amount of energy is saved and speed of cluster formation is increased. We proposed an efficient clustering approach by combining good features of LEACH approach and fuzzy logic (with little computation overhead).

We compared our method with three similar methods, LEACH and LEACH-FL in lifetime, energy consumption and number of death of nodes. Simulation output with Matlab shows that our method saves more energy, thus increases lifetime of WSNs.

\section{References}

[1] K. Akkaya, M. Younis, "A Survey of Routing Protocols in Wireless Sensor Networks," Ad Hoc Network Journal, Vol. 3/3, pp. 325-349, 2005.
[2] J. N. Al-Karak and A. E.Kamal, "Routing techniques in wireless sensor network: A survey," IEEE wireless communications, Vol. 11, pp. 6-28, 2004.

[3] I. F. Akyildiz, W. Su, Y. Sankarasubramaniam, and E. Cayirci, "Wireless sensor networks: a survey," Computer Networks, Vol. 38, pp. 393- 422, 2002.

[4] J. Breckling, Ed., The Analysis of Directional Time Series: Applications to Wind Speed and Direction, ser. Lecture Notes in Statistics. Berlin, Germany: Springer, vol. 61, 1989.

[5] Seyyed Jalaleddin Dastgheib, Hamed Oulia, Mohammad Reza Sadeqi Ghassami, An Efficient Approach for Clustering in Wireless Sensor Network Using Fuzzy Logic, International Conference on Computer Science and Network Technology, Harbin, China 24-26, IEEE, 2011

[6] I. Gupta, D. Riordan and S. Sampalli, "Cluster-head Election using Fuzzy Logic for Wireless Sensor Networks," In Proceedings of the 3rd Annual Communication Networks and Services Research Conference, Washington, DC, USA, pp.255-260, 2005.

[7] J. Myoung Kim, S. Park, Y. Han and T. Chung, "CHEF: Cluster Head Election mechanism using Fuzzy logic in Wireless Sensor Networks," 10th International Conference on Advanced Communication (ICACT),Gangwon-Do,South_Korea, pp.654-659, 2008.

[8] D. De, A Distributed Algorithm for Localization Error Detection-Correction, Use in In-network Faulty Reading Detection:Applicability in Long-Thin Wireless Sensor Networks, in Conference IEEE 2009.

[9] Heinzelman, A. Chandrakasan and H. Balakrishnan, An application-specific protocol architecture for wireless microsensor networks, in IEEE Transactions on Wireless communications, pp. 660 - 670, 2002. 
[10] G. W. Nurcahyo, Selection of Defuzzification Method to Obtain Crisp Value for Representing Uncertain Data in a Modified Sweep Algorithm, Journal of Computer Science \& Technology (JCS\&T), Vol. 3 No. 2, October 2003.

[11] F. Vanheel, J. Verhaevert, "Automated Linear Regression Tools Improve RSSI WSN Localization in Multipath Indoor Environment," EURASIP Journal on Wireless Communications and Networking, vol.2011/2011,pp.1-27, 2011.

[12] http://www.mathworks.com/FuzzyLogic Toolbox user's guide.

[13] Z. Qin, M. Bai and D. Ralescu, A fuzzy control system with application to production planning problems, Information Sciences Elsevier Volume 181, Issue 5, Pages 1018-1027, 2011

[14] G. C. D. Sousa and B. K. Bose. "A FUZZY Set Theory Based Control of a Phase-Controlled Converter DC Machine Drive", 1991 IEEE hi.App. Sot. Annu. Meeting, pp.854-861, 1991.
[15] D. Z. Šaletić, D. Velašević: The formal description of a rule based fuzzy expert system, Proceedings of Symposium on Computer Scences and Information Technologies, Kopaonik 27-31. III 2000, pp. $251-220$, YUiNFO 2000

[16] W. Van Leekwijck, E.E. Kerre:Defuzzification: criteria and cla sification, Fuzzy Sets and Systems, 108, pp. 159-178,1999

[17] Dragan Z. Saletic, Dusan M. Velasevic, Nikos E. Mastorakis, "Analysis of Basic Defuzzification Techniques" inRecent Advances in Computers, Computing and Communications, pp. 247-252, WSEAS Press, 2002.

[18] E. H. Mamdani, "Application of fuzzy logic to approximate reasoning using linguistic synthesis," IEEE Transactions on Computers, Vols. C-26, pp. 1182-1191, 1977. 